\title{
The Drawback of Image Resizing Based on Seam Carving
}

\author{
Zijuan Zhang \\ Shaanxi Polytechnic Institute, Xianyang, 712000, China \\ Email address: bluesky1023@126.com
}

Keywords: seam carving; backward algorithm; forward; image resizing

\begin{abstract}
Lots of image resizing algorithms that based on seam carving belong to the discrete resizing methods. Seam-carving algorithms refer to delete or insert pixel lines in order to resize image's size. Pixel lines horizontally or vertically pass through the whole image, deleting the pixel line to make the reduction, copying the pixel line to make the magnification, processing level (vertical) pixel line could change the height (width) of image. In order to keep the main characteristic content in image, many seam carving algorithms delete or insert pixel line that has the smallest energy each time. Lots of seam carving algorithms can scale the size of image, protect the important content in the original image, achieve good effect about adaptation, but these algorithms will produce many problems, such as deleting distortion excessively.
\end{abstract}

\section{Introduction}

On the Siggraph conference which was held in 2007, Avidan and Shamir [1] put forward an image resizing algorithm based on seam carving method, this new way is also known as Backward Seam Carving(BSC) algorithm. Seam carving refers to the algorithm that inserts or removes pixel seams in order to scale image, these algorithms delete or insert the pixels line which have minimum energy and realize image resizing which can protect the important content in the original image better, achieve the good effect with regard to resizing effect. After the BSC algorithm was put forward, many scholars propose retargeting methods[2-4] based on seam carving.

\section{Image resizing algorithm based on seam carving Method}

In the reference [1], the content's importance degree is calculated by gradient values about pixels. Backward Seam Carving algorithm is one of the most original algorithms that were based on seam carving. Figure 1 shows a BSC algorithm example, figure 1 (a) is the original image, a vertical and a horizontal seam, the pixels seam is a connected path which has low energy. Figure 1 (b) uses the gradient map in order to represent the original image's energy (also regarded as the importance degree map). Figure 1 (c) (d) are the vertical, horizontal path picture respectively which is presented to calculate pixels seam path. BSC method automatically deletes horizontal pixel seam path or inserts into the vertical pixel path in order to implement image resizing, figure 1 (e) is the result of the original image after BSC algorithm. Figure 1 (f) is the result after using unified scale (it is also can be known as Scaling, SCL). Compared with the traditional scaling algorithm, BSC algorithm achieves image resizing and protects the source image's important content better.

In order to define the image's importance degree about pixels, BSC algorithm regards image gradient norm as the energy function in its original literature. Supposing $I$ is the original image, then the energy function can be shown

$$
e(I)=\left|\frac{\partial}{\partial x} I\right|+\left|\frac{\partial}{\partial y} I\right|
$$

The energy function's value represents the pixels’ importance degree. Supposing the image's size is $m \times n, m$ represents column, $n$ represents row. Then the vertical pixels seam path $s^{v}$ can be 
represented by

$$
s^{v}=\left\{s_{i}^{v}\right\}_{i=1}^{n}=\{(x(i), i)\}_{i=1}^{n}, \text { s.t. } \forall i,|x(i)-x(i-1)| \leq 1
$$

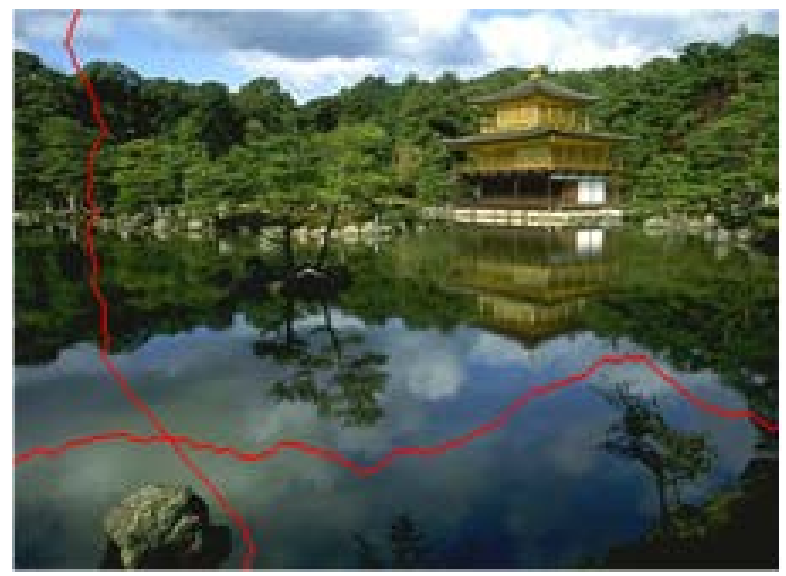

(a) original image and seams (red)

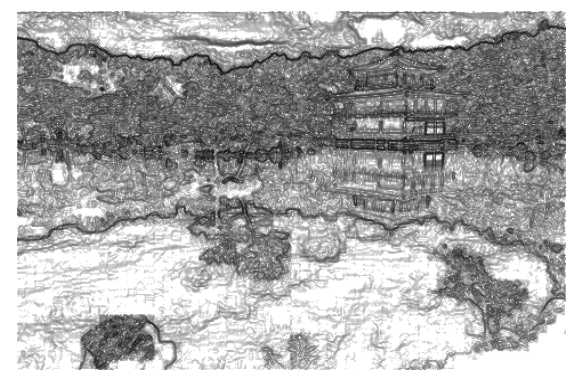

(b) gradient map



(c) vertical path

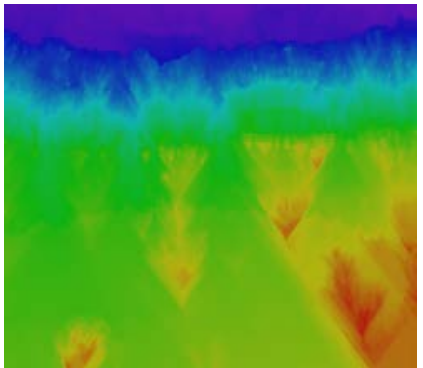

(d) horizontal path

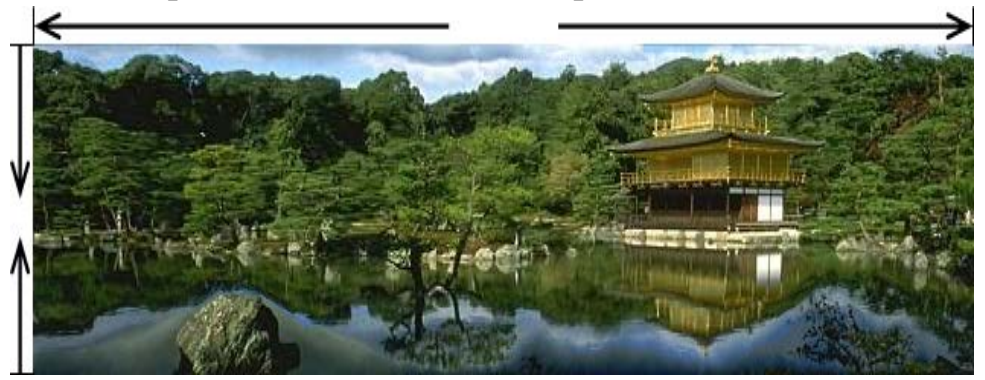

(e) the retargeting result by BSC

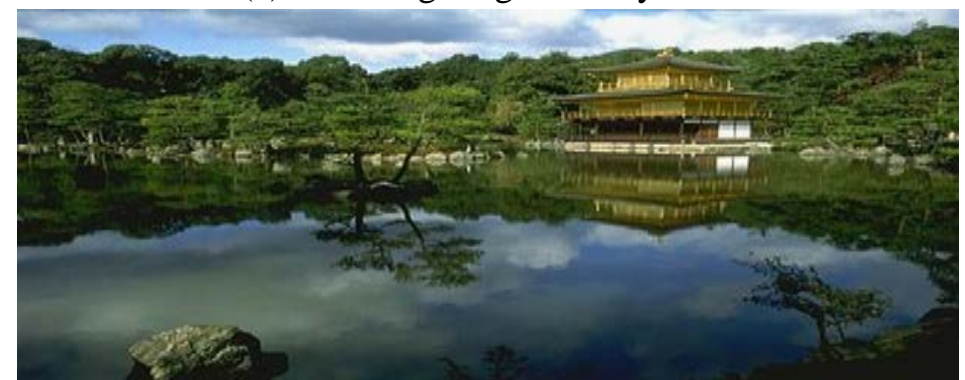

(f) the resizing result by SCL

Figure1. The example of BSC algorithm[1]

$x(i)$ can be seen as a mapping: $x:[1, \cdots, n] \rightarrow[1, \cdots m]$. That is to say, vertical pixel seam line is a 8 connected pixels path from the first row to the last row in original image, and each row in the 
original image has only one pixel in every line. If $y$ is mapping: $y:[1, \cdots m] \rightarrow[1, \cdots, n]$, then the horizontal seam row $s^{h}$ can be seen as

$$
s^{h}=\left\{s_{j}^{h}\right\}_{j=1}^{m}=\{(j, y(j))\}_{j=1}^{m}, \text { s.t, } \forall j,|y(j)-y(j-1)| \leq 1
$$

The pixels on vertical seam path $s$ is

$$
I_{s}=\left\{I\left(s_{i}\right)\right\}_{i=1}^{n}=\{I(x(i), i)\}_{i=1}^{n}
$$

It is very important to note that if vertical (horizontal) seam line is removed from the original image, pixels on the seam's right side should be moved to left, in order to make up the gap which is due to deleting.

Based on energy function, the cost about vertical seam path $E(s)$ can be defined by

$$
E(s)=E\left(I_{s}\right)=\sum_{i=1}^{n} e\left(I\left(s_{i}\right)\right)
$$

Minimizing the cost of seam in order to search the optimal seam $s^{*}$

$$
s^{*}=\min _{s} E(s)=\min _{s} \sum_{i=1}^{n} e\left(I\left(s_{i}\right)\right)
$$

Then, we could use dynamic program algorithm for searching the optimal seam. For each pixel's position $(i, j)$ on image, the cumulative energy $M(i, j)$ is defined by

$$
M(i, j)=e(i, j)+\min (M(i-1, j-1), M(i, j-1), M(i+1, j-1))
$$

In order to find the optimal vertical seam path, we should traverse all pixels from the second row to the last row in original image firstly and then calculate the accumulated energy value. At the end in this process, the accumulated energy about minimum point in last row is the optimal vertical seam on the end. Then we could come back from that point, searching the cumulative minimum energy pixel's location and continuing to back from this place which was regarded as the new starting point, continuing to search it in the same manner, repeating this iterative process until the first line in the original image to search the optimal path pixel with vertical path. The definition of the optimal horizontal pixel seam path is also calculated by a similar process.

Literature [5] has focused on that Backward algorithm only considers the pixel's energy itself, presented the Forward Seam Carving(FSC) algorithm. This method increases the energy that due to deleting the pixels which could make the new adjacent pixels, implements a little improvement on BSC method. Due to the FSC algorithm considers the deleting pixel path that makes other pixels to produce new adjacent relations and increase energy value, comparing to the BSC method, FSC algorithm can achieve better result. Additionally, the literature [6-7] have proposed different improved algorithms about seam carving.

\section{Summary}

Although the appearance about many retargeting methods based on BSC algorithm can obtain the better scaling effect, as the original seam carving algorithm, the status about the BSC method is without a doubt, its innovation and pioneer are unmatched with regard to other methods. At the same time in a variety improved algorithms that based on BSC algorithm, the effect about Forward Seam Carving algorithm is significant and could eliminate artificial noise points effectively. Whenever 
BSC, FSC or other improved algorithms which based on seam carving, they are discrete resizing methods which were based on pixel level and will produce distortion problem, such as deleting distortion excessively.

\section{References}

[1] S. Avidan, A. Shamir, Seam carving for content-aware image resizing, J. ACM Transactions on Graphics. 26(3) (2007) 1-10

[2] J.C. Yoon, S.Y. Lee, I.K. Lee, Optimized image resizing using flow-guided seam carving and an interactive genetic algorithm, in Multimedia Tools and Applications, 2014, pp. 1013-1031.

[3] M. Kazu, I. Masaaki, Seam merging for image resizing with structure preservation, in 2011 IEEE International Conference on Acoustics, Speech and Signal Processing, 2011, pp. 1001-1004

[4] J.D. Wei, Y.J. Lin, Wu Y.J, A patch analysis method to detect seam carved images, J. Pattern Recognition Letters, 36 (2014) 100-106

[5] M. Rubinstein, A. Shamir, S. Avidan, Improved seam carving for video retargeting, J. ACM Transactions on Graphics, 27(3) (2008). 1-9

[6] H. Nicholas, E. Parisa, Seam carving for enhancing image usability on mobiles, in Proceedings of the 22nd British HCI Group Annual Conference on HCI, 2008, pp. 131-134

[7] N. Ryo, U. Kei, T. Keita Stereo image retargeting with shift-map, J. IEICE Transactions, 94-D(6), (2011). 1345-1348 